\title{
EQUIPE EDITORIAL E AVALIADORES AD HOC
}

\section{EDITORA-CHEFE}

Dra. Greice da Silva Lorenzzetti Andreis

Instituto Federal de Educação, Ciência e Tecnologia do Rio Grande do Sul (IFRS), Campus Caxias do Sul, RS, Brasil

\section{EDITORA ADJUNTA}

Dra. Katia Arcaro

Instituto Federal de Educação, Ciência e Tecnologia do Rio Grande do Sul (IFRS), Campus Caxias do Sul, RS, Brasil

\section{COMISSÃO EDITORIAL}

Ma. Daiane Scopel Boff

Instituto Federal de Educação, Ciência e Tecnologia do Rio Grande do Sul (IFRS), Campus Caxias do Sul, RS, Brasil

\section{Me. Henri Luiz Fuchs}

Instituto Federal de Educação, Ciência e Tecnologia do Rio Grande do Sul (IFRS), Campus Bento Gonçalves, RS, Brasil

Dra. Katia Arcaro

Instituto Federal de Educação, Ciência e Tecnologia do Rio Grande do Sul (IFRS), Campus Caxias do Sul, RS, Brasil

Me. Michelsch João da Silva

Instituto Federal de Educação, Ciência e Tecnologia de Santa Catarina (IFSC), Campus Florianópolis, SC, Brasil

\section{Dr. Nicolau Matiel Lunardi Diehl}

Instituto Federal de Educação, Ciência e Tecnologia do Rio Grande do Sul (IFRS), Campus Canoas, RS, Brasil

\section{Dr. Rodrigo Sychocki da Silva}

Universidade Federal do Rio Grande do Sul (UFRGS), Instituto de Matemática e Estatística (IME), Porto Alegre, RS, Brasil 


\title{
CONSELHO EDITORIAL CONSULTIVO
}

\author{
Dra. Adriana Breda \\ Universitat de Barcelona, Barcelona, Espanha \\ Dr. Carloney Alves de Oliveira
}

Universidade Federal de Alagoas (UFAL), Centro de Educação (CEDU), Campus Aristóteles Calazans Simões, Maceió, AL, Brasil

Dra. Carolina Augusta Assumpção Gouveia

Centro de Ensino Superior de Valença (CESVA/FAA), Valença, RJ, Brasil

\section{Dra. Franciele Corti}

Universitat de Barcelona (UB), Campus Mundet, Barcelona, Espanha

\section{Dr. Francisco Regis Vieira Alves}

Instituto Federal de Educação, Ciência e Tecnologia do Estado do Ceará (IFCE), Fortaleza, CE, Brasil

Dra. Maria Cristina Caminha de Castilhos França

Instituto Federal de Educação, Ciência e Tecnologia do Rio Grande do Sul (IFRS), Campus Porto Alegre, RS, Brasil

\author{
Dr. Moiseis Cecconello \\ Universidade Federal de Mato Grosso (UFMT), Cuiabá, MT, Brasil \\ Dr. Rene Carlos Cardoso Baltazar Júnior \\ Universidade Federal do Rio Grande (FURG), Rio Grande, RS, Brasil \\ Dr. Wagner Marcelo Pommer \\ Universidade Federal de São Paulo (UNIFESP), Campus Diadema, SP, Brasil
}

\section{EDITORES DE TEXTO}

\section{Dr. Claudionor Ferreira Araujo}

Instituto Federal de Educação, Ciência e Tecnologia do Rio Grande do Sul (IFRS), Campus Caxias do Sul, RS, Brasil

\author{
Dra. Cristina Maria Pescador \\ Universidade de Caxias do Sul (UCS), Caxias do Sul, RS, Brasil
}

Dra. Silvana Kissmann

Instituto Federal de Educação, Ciência e Tecnologia do Rio Grande do Sul (IFRS), Campus Caxias do Sul, RS, Brasil

\section{APOIO TÉCNICO}

Srta. Camila Gasparin Magnaguagno

Instituto Federal de Educação, Ciência e Tecnologia do Rio Grande do Sul (IFRS), Bolsista BICTES/IFRS, Campus Caxias do Sul, RS, Brasil 


\title{
AVALIADORES AD HOC
}

\author{
Dr. Alvino Alves Sant'Ana
}

Universidade Federal do Rio Grande do Sul (UFRGS), Porto Alegre, RS, Brasil

Dra. Ana Carolina Costa Pereira

Universidade Estadual do Ceará (UECE), Fortaleza, CE, Brasil

Dr. Antonio Carlos Brolezzi

Universidade de São Paulo (USP), Instituto de Matemática e Estatística (IME), São Paulo, SP, Brasil

Me. Carlos Bino de Souza

Instituto Federal de Educação, Ciência e Tecnologia de Pernambuco (IFPE), Campus Pesqueira, PE, Brasil

Me. Cassiano Scott Puhl

Pontifícia Universidade Católica do Rio Grande do Sul (PUC/RS), Porto Alegre, RS, Brasil

Dra. Celina Aparecida Almeida Pereira Abar

Pontifícia Universidade Católica de São Paulo (PUC/SP), São Paulo, SP, Brasil

Dr. Dassael Fabrício dos Reis Santos

Instituto Federal de Educação, Ciência e Tecnologia Goiano (IF Goiano), Campus Urutaí, Urutaí, GO, Brasil

\section{Dr. David Antonio da Costa}

Universidade Federal de Santa Catarina (UFSC), Santa Catarina, SC, Brasil

Dra. Franciele Corti

Universitat de Barcelona (UB), Campus Mundet, Barcelona, Espanha

Ma. Isabelle Coelho da Silva

Instituto Federal de Educação, Ciência e Tecnologia do Ceará (IFCE), Programa de Pós-Graduação em Ensino de Ciências e Matemática (PGECM), Fortaleza, CE, Brasil

Me. Jeremias Stein Rodriguês

Instituto Federal de Educação, Ciência e Tecnologia de Santa Catarina (IFSC), Florianópolis, SC, Brasil

$$
\text { Dr. João Cândido Moraes Neves }
$$

Instituto Federal de Educação, Ciência e Tecnologia do Rio Grande do Sul (IFRS), Campus Caxias do Sul, RS, Brasil

\section{Me. Jonas da Conceição Ricardo}

Universidade Estácio de Sá (UNESA), Santa Cruz, RJ; Secretaria de Estado de Educação do Rio de Janeiro (SEEDUCRJ), Rio de Janeiro, RJ; Centro Federal de Educação Tecnológica Celso Suckow da Fonseca (CEFET/RJ), Rio de Janeiro, RJ, Brasil

Dra. Karin Ritter Jelinek

Universidade Federal do Rio Grande (FURG), Rio Grande, RS, Brasil

$$
\text { Dr. Mateus Alegri }
$$

Universidade Federal de Sergipe (UFS), Aracaju, SE, Brasil 


\title{
Me. Nitiele Medeiros Contessa
}

Instituto Federal de Educação, Ciência e Tecnologia do Rio Grande do Sul (IFRS), Campus Caxias do Sul, RS, Brasil

\author{
Dr. Oclide José Dotto \\ Caxias do Sul, RS, Brasil
}

\section{Dra. Paula Reis de Miranda}

Instituto Federal de Educação, Ciência e Tecnologia do Sudeste de Minas Gerais (IF Sudeste MG), Campus Rio Pomba, Departamento Acadêmico de Matemática, Física e Estatística, Rio Pomba, MG, Brasil

\author{
Dr. Rene Carlos Cardoso Baltazar Júnior \\ Universidade Federal do Rio Grande (FURG), Rio Grande, RS, Brasil
}

\author{
Dra. Rúbia Emmel \\ Instituto Federal de Educação, Ciência e Tecnologia Farroupilha, Campus Santa Rosa, RS, Brasil
}

Ma. Sabrina Arsego Miotto

Instituto Federal de Educação, Ciência e Tecnologia do Rio Grande do Sul (IFRS), Campus Caxias do Sul, RS, Brasil

Ma. Sandra Denise Stroschein

Instituto Federal de Educação, Ciência e Tecnologia do Rio Grande do Sul (IFRS), Campus Bento Gonçalves, RS, Brasil

\section{Dra. Suzete Maria Silva Afonso}

Universidade Estadual Paulista (UNESP), Campus de Rio Claro, Rio Claro, SP, Brasil

\section{Ma. Vânia Luisa Behnen Cord}

Instituto Federal de Educação, Ciências e Tecnologias do Rio Grande do Sul (IFRS), Campus Ibirubá, Ibirubá, RS, Brasil

\author{
Ma. Vânia Maria Pinheiro Slaviero
}

Universidade de Caxias do Sul (UCS), Caxias do Sul, RS, Brasil

\author{
Dr. Wagner Marcelo Pommer \\ Universidade Federal de São Paulo (UNIFESP), Campus Diadema, SP, Brasil
}

